\title{
ON THE ANALOG FOR DIFFERENTIAL EQUATIONS OF THE HILBERT-NETTO THEOREM
}

\author{
BY H. W. RAUDENBUSH, JR.
}

The Hilbert-Netto theorem for polynomials* has an analog, obtained by J. F. Ritt, $\uparrow$ for differential polynomials. $\ddagger$ By a differential polynomial is meant a polynomial in a finite number of unknown functions $y_{1}, \cdots, y_{n}$ of the independent variable $x$ and a certain number of their derivatives. To any finite set of differential polynomials $F_{1}, \cdots, F_{r}$ there corresponds a finite system of ordinary algebraic differential equations $F_{1}=0, \cdots, F_{r}=0$. It is supposed that the coefficients of the $F_{i}$ are all elements of a field $₹$ of differentiable functions of $x$ which is closed with respect to differentiation. Let $G$ be any differential polynomial with coefficients in $₹$ such that $G=0$ has every solution $\S$ of the system. If we denote by $\Sigma$ the totality of differential polynomials that are linear combinations of the $F_{i}$ and a certain number of their derivatives with differential polynomials with coefficients in $\mathfrak{F}$ as coefficients, then Ritt's theorem is that some power of $G$ is in $\Sigma$.

We show in the following that this is as far in this direction as the analogy between the theories of polynomials and differential polynomials extends. For our result is that, contrary to the easy conjecture, it is not generally true that for a given system there exists a single positive integer $\rho$ such that any differential polynomial $G$, defined as above for that system, has the power $G^{\rho}$ in the corresponding $\Sigma$. We give an example of a system for which there exist $G$ 's with arbitrarily high powers not in $\Sigma$. This result shows, in particular, why the ideal theory of

* See B. L. van der Waerden, Moderne Algebra, vol. 2, p. 66.

$\dagger$ J. F. Ritt, Differential Equations from the Algebraic Standpoint, Colloquium Publications of this Society, vol. 14. (Cited as $\alpha$.)

$\ddagger$ J. F. Ritt, Systems of algebraic differential equations, Annals of Mathematics, (2), vol. 36 (1935), p. 293. The term "differential polynomial" is equivalent to the terms "differential form" or "form" used in $\alpha$.

$\S$ See $\alpha$ for a precise definition. For an abstract formulation, see H. W. Raudenbush, Jr., Ideal theory and algebraic differential equations, Transactions of this Society, vol. 36 (1934), pp. 361-368. 
differential polynomials as far as it has been developed is marked by the absence of primary ideals.*

In our example, the finite system consists of the single equation $F=y^{3}=0$. The differential polynomials $G$, such that $G=0$ has every solution of $F=0$, are simply those differential polynomials in $y$ that have no term free of $y$ and its derivatives. We suppose that there exists a single positive integer $\rho$, such that for any $G$, the power $G^{\rho}$ is in $\Sigma$, and show that this assumption leads to a contradiction.

The weight of a power product in $y$ and a certain number of its derivatives is defined as the sum of the products of the order of each derivative present and the degree to which it appears. The totality of differential polynomials whose terms are all of degree $\rho$ and weight $w$, where $w$ is to be fixed later, we shall denote by $\Sigma_{w}^{\rho}$.

A linear basis with respect to $\mathfrak{F}$ for $\Sigma_{w}^{\rho}$ may be obtained by taking all products consisting of $F$ or some one of its derivatives $d^{i} F / d x^{i}, i \leqq w$, multiplied by a power product in $y$ and its derivatives of degree $\rho-3$ and weight $w$ or $w-i$, respectively. The number of differential polynomials in a set of linearly independent differential polynomials of $\Sigma_{w}^{\rho}$ is consequently not greater than the number of distinct power products of $y$ and its derivatives of degree $\rho-3$ and weight $w$ or less. An upper bound to the number is $(w+1)^{\rho-3}+w^{\rho-3}+(w-1)^{\rho-3}+\cdots+1$, or consequently and more simply, $(w+1)^{\rho-2}$.

We consider now the power products in $y$ and its derivatives of degree $\rho$ and weight $w$. Suppose, for simplicity, that $w$ is divisible by $\rho$. Then their number is greater than $w^{\rho-1} /\left(\rho^{\rho-1}(\rho-1) !\right)$. This lower bound is obtained by noticing that if we fill the first places in the product with $y$ or any of its derivatives of order less than $w / \rho$, then it is always possible to fill the last place with a derivative of order greater than $w / \rho$ to make a power product of degree $\rho$ and weight $w$. The number of permutations with repetitions of the $w / \rho$ things ( $y$ and its derivatives of order less than $w / \rho)$ taken $\rho-1$ at a time is $(w / \rho)^{\rho-1}$. Not more than $(\rho-1)$ ! of these permutations can give a single combination, that is, a single power product. Now since these power products form a linearly independent set with re-

\footnotetext{
* See Raudenbush, loc. cit.
} 
spect to $\mathfrak{F}$ and since for a certain large $w$, their number exceeds $(w+1)^{p-2}$, we conclude that, for a certain large $w, \Sigma_{w}^{p}$ does not contain all power products of degree $\rho$ and weight $w$.

Under our assumption, on the other hand, $\Sigma$ would contain all differential polynomials of the form $\left(a_{0} y+a_{1} d y / d x+\ldots\right.$ $\left.+a_{w} d^{w} y / d x^{w}\right)^{\rho}$, where the $a_{i}$ are arbitrary elements of $\mathfrak{F}$. It is easily seen that each power product of degree $\rho$ and weight $w$ is a linear combination of certain of these differential polynomials and hence is in $\Sigma_{w}^{\rho}$. This contradiction shows that for the system $F=y^{3}=0$ no integer $\rho$ exists or, in other words, differential polynomials $G$ exist having arbitrarily large powers not in $\Sigma$.

YALE UNIVERSITY

\section{NOTE ON THE GALERKIN AND PAPKOVITCH STRESS FUNCTIONS}

BY R. D. MINDLIN

1. Introduction. H. M. Westergaard* has given a useful interpretation of the Galerkin stress functions $\dagger$ as the components of a vector function satisfying a fourth order equation. From the Galerkin vector, P. F. Papkovitch $\ddagger$ has developed a new solution of the three-dimensional elasticity equations for a homogeneous, isotropic solid in terms of harmonic functions. The same solution has been given by H. Neuber. $\$$

Some interesting aspects of the Galerkin and Papkovitch functions may be observed when they are approached from a consideration of Helmholtz's theorem. In so doing, it is found that these functions may be reached by a direct analytical process and that they are connected through simple functional rela-

* H. M. Westergaard, this Bulletin, vol. 41 (1935), p. 695.

$\dagger$ B. Galerkin, Comptes Rendus, vol. 190 (1930), p. 1047. See also Todhunter and Pearson, History of Elasticity, vol. 2, part 2, pp. 268-270.

‡P. F. Papkovitch, Comptes Rendus, vol. 195 (1932), pp. 513, 754. J. N. Goodler calls attention to Todhunter and Pearson, loc. cit., vol. 2, part 2, p. 373 .

$\S$ H. Neuber, Zeitschrift für angewandte Mathematik und Mechanik, vol. 14 (1934), p. 203. 